\title{
Quantitative inspection of thickness of thermally sprayed coatings by flash pulse thermographic method
}

\author{
by M. Švantner ${ }^{1}$, L. Muzika ${ }^{1}$ and A. Moskovchenko ${ }^{1,2}$, Šárka Houdková1 ${ }^{1}$ Petra Frková ${ }^{3}$ \\ ${ }^{1}$ University of West Bohemia, New Technologies - Research Center, Univerzitní 8, Plzeň, Czechia, \\ msvantne@ntc.zcu.cz, muzika@ntc.zcu.cz, moskovchenko.alexey@gmail.com, houdkov@ntc.zcu.cz \\ 2 Tomsk Polytechnic University, Lenin Avenue 30, Tomsk, Russia \\ ${ }^{3}$ Research and Testing Institute PIzeň, Tylova 1581/46, PIzeň, Czechia, frkova@vzuplzen.cz
}

\begin{abstract}
Flash-pulse thermography is a method for a detection of discontinuities or inhomogeneities in materials at their surface. It is based on an excitation of an inspected sample by a short pulse and analysis of its thermal response. An application of flash-pulse thermography for a quantitative inspection of thickness differences of thermally sprayed coatings is introduced in this contribution. Requirements for a synchronization of thermal response recording and data smoothing precision are described. Signal derivative, pulse-phase and time power-transformation (P-function) methods for an evaluation of the thermographic records are presented. Procedure and results of the inspection are demonstrated on HVOF, TWAS and flame sprayed coatings. A comparison of the method showed that the P-function method is the most suitable for a quantification of coating thickness differences. Some characteristic of the method are demonstrated by numerical computation.
\end{abstract}

\section{Introduction}

Thermographic testing [1] is an active thermography method, which is used for a near-surface material inspection. The research in this contribution is focused on a quantitative evaluation of a coating thickness by a flash pulse thermography. This method is based on an external excitation by a short pulse, which induces a thermal process inside an inspected object. Material inhomogeneities near the tested surface affect the process and these inhomogeneities then can be detected by an analysis of a thermal response of the object. A thermal response of an excited object is mostly evaluated by one of the standard methods, which are, for example, signal derivation, pulse-phase evaluation or thermographic signal reconstruction. The pulse thermography is introduced more in detail, for example, in [2] or [3].

Coatings thickness and its homogeneity is one of important parameters of the coatings. It is a quality parameter and it can influence mechanical, fatigue or other properties of the coatings. Traditional methods for a non-destructive measurement of coating thickness are based on electro-magnetic or ultrasonic principles. These measurements are mostly performed using a local probe. If a measurement of surface thickness inhomogeneity is required, then a scanning over the whole surface has to be done. A benefit of the thermographic methods is that they allow an area inspection. Basic results of a thermographic inspection are indications, which bring information about an occurrence and position of inhomogeneities without any additional quantification. Extended evaluation methods are developed for a quantification of thermographic inspection results, as for example a depth of found defects or a thickness of inspected coatings. To these methods belong, for example, an evaluation of Contrast to Noise Ratio [4]. These methods, however, allow a quantification of an intensity of the indications only. Thus, they are mostly useful for an evaluation of a detectability, but they are not usable for a quantitative IRNDT inspection.

Evaluation procedures resulting in a numerical quantification of thermographic inspection results were a topic of a research described in this contribution. The contribution deals with flash-pulse thermography, which was used for an inspection of thickness differences of thermally sprayed coating. Samples made by three different coating technologies were examined by a flash pulse thermographic measurement. A response of the thermographic measurements was evaluated by different methods and a sensitivity of an output quantity to changes of a thickness of the tested coatings were analysed. The goal of the research was to select a measurement and evaluation procedure, which could provide results with a high sensitivity to coating thickness changes, but which could be robust enough and resistant to influence of other measurement parameters. With this goal, an influence of parameters of coatings and/or evaluation procedure is discussed and some features are demonstrated by a numerical computation results.

\section{Experiment description}

Thermally sprayed coatings were inspected by a flash pulse thermographic method and the measured thermal response results were evaluated by different methods. The coatings were deposited on a steel substrate. Three types of coatings were investigated: Fe-Cr coatings made by a Twin Wire Arc Spraying technology, Sn-Sb-Cu coatings made by Flame Spraying technology and WC-CoCr coatings made by High Velocity Oxygen Fuel spraying technology. Samples 
with step-increasing coatings thickness in a rage from 200 to 600 um were made for each material / technology. The surfaces of the coatings were left in the as-sprayed state, without any subsequent treatment.

Each sample was inspected using the same testing configuration. A flash lamp with a pulse length about $6 \mathrm{~ms}$ and the maximum power $6 \mathrm{~kJ}$ was used for an excitation. A thermal response was recorded by a high-speed cooled InSb based detector thermographic camera with framerate $250 \mathrm{~Hz}$. A synchronization between the flash lamp and the camera was realized by a hardware unit, which started a recording and initiated a flash pulse. Three evaluation methods were applied on recorded thermal responses: FFT based processing (phase evaluation), time-derivation based processing and a time-power transformation based method (P-function). The time-derivation method was based on an approximation of a temperature time curve in each pixel by a suitable polynomial function and a performing of $1^{\text {st }}$ time-derivative of this function. The P-function method makes a signal transformation in each pixel, which leads to an occurrence of a local minimum on a transformed curve. Then a position of the minimum point on a time axis is an evaluated quantity. Basic principles of this method are described, for example, in [5]. The results of all methods were quantitatively evaluated in a form of a difference to an initial value (raw signal, FFT, time-derivation) or as a time of the minimum (P-function).

\section{Results}

The experiments showed differences of about $20 \%$ between results of repeated measurements of the same sample at the same conditions. Such differences were comparable with differences caused by a change of a thickness of the tested coatings. It was revealed that this lack of repeatability was related to a synchronization of a flash pulse excitation and a thermographic data recording. The flash pulse peak occurred at different positions between two subsequent recorded frames if a standard thermographic testing configuration was used. Thus, changes of the experimental configuration related to a synchronization of the flash lamp and the infrared camera were made, which resulted to a repeatability of measurements about $98.8 \%$ (i.e. differences $1.2 \%$ ).

The experiment showed that the sensitivity of the tested evaluation methods to a change of the coating thickness of about $0.1 \mathrm{~mm}$ is in a range from a few percent to about $200 \%$. The best results brought the P-function method - the evaluation quantity increased of about $600 \%$ for the Fe-Cr coating when the coating thickness increased from 0.22 to $0.59 \mathrm{~mm}$. A sensitivity of the other evaluation methods was up to $160 \%$ for this coating. It was determined that results of other methods are highly sensitive to other measurement parameters and it was quite complicated to arrange a repeatable measurement procedure. An output-sensitivity to thickness differences of the P-function method was significantly lower for other coatings. That was about $20 \%$ for the Sn-Sb-Cu coating and $60 \%$ for the WC-CoCr coating. These differences were connected with different diffusivity of the tested coatings. An influence of a coatings diffusivity, which can cause significant changes of results or, in some cases, to a necessity of an adjustment of evaluation parameters, was also verified by a numerical computation results.

\section{Conclusions}

The research showed a critical influence of a synchronization of a pulse excitation with a thermographic recording. This issue was solved by an improvement of hardware/software configuration set-up, which led to repeatability about $98.8 \%$. It was determined that the P-function method outputs had the highest sensitivity to the changes of the thickness of the tested coatings. The output signal changes were from 20 to $600 \%$ based on a coating material. This method was also more stable, i.e. more resistive to an influence of other measurement parameters, than the derivative based or FFT based methods. However, as it was not a physical model approach, thus, the results were still dependent on an experimental setup configuration and coatings thermal properties. This leads to a necessity of a calibration procedure for each measurement at this state of development.

Funding: The work was supported by ERDF project "LABIR-PAV / Pre-application research of infrared technologies" Reg. No. CZ.02.1.01/0.0/0.0/18_069/0010018 by the project SGS-2019-008.

\section{REFERENCES}

[1] Maldague, X.P. V. Theory and practice of infrared technology for nondestructive testing; Wiley series in microwave and optical engineering; Wiley, 2001; ISBN 9780471181903.

[2] Balageas, D.L. Defense and illustration of time-resolved pulsed thermography for NDE. QIRT J. 2012, 9, 3-32.

[3] Maldague, X.; Galmiche, F.; Ziadi, A. Advances in pulsed phase thermography. Infrared Phys. Technol. 2002, $43,175-181$

[4] Švantner, M.; Muzika, L.; Chmelík, T.; Skála, J. Quantitative evaluation of active thermography using contrastto-noise ratio. Appl. Opt. 2018, 57, D49-D55

[5] Vavilov, V.P.; Pawar, S.S. A novel approach for one-sided thermal nondestructive testing of composites by using infrared thermography. Polym. Test. 2015, 44, 224-233. 